\title{
TRANSMISSIONS WITH THREE POWER-FLOWS FOR TRUCKS AND TRACTORS
}

\author{
Konstantin Salamandra \\ Mechanical Engineering Research Institute of the Russian Academy of Sciences, Russia \\ ksalamandra@yandex.ru
}

\begin{abstract}
Trucks, agricultural machinery and tractors are equipped with transmissions consisting of main 3speed or 4-speed gearbox with fixed axles of gears and a planetary splitter and/or a planetary range change unit. These planetary gear sets reduce the rotation speed or work in direct gear depending on the driving conditions of the machine. The splitter and range change unit are also providing up to 4 times extension of the number of gear ratios realized by transmission. If the planetary gear set in such transmissions will work as differential drive, it will allow increasing the number of gear ratios in the combined planetary-layshaft transmissions and the number of operating regimes of the tractor consequently. As a result, the gears with fixed axles can work separately in one-stream operation mode of the planetary gear set (reducer or direct gear) and in a two-stream mode - in parallel with other layshaft gears, when the planetary gear set is working as a differential drive. It was shown that combined two-stream planetary-layshaft transmissions consist of simple gear trains, have high efficiency and provide more overall gear ratios, if compared with transmissions consisted of the gear trains of one type. Two planetary gear sets operating as differential drives increase the number of parallel power-flows to three, which allow further improvements of the functionality of transmissions. In addition, if in two-stream transmissions 50$60 \%$ of the input power is transmitted along the paths, then in three-stream transmissions this percentage is reduced by a factor of 1.5 or 2 . The purpose of the paper is to analyse structural schemes of planetary-layshaft transmissions with three power-flows, to determine their general characteristics and to develop a method for synthesis of the kinematic diagrams of these transmissions.
\end{abstract}

Keywords: transmission, synthesis, planetary gear, gear with fixed axle, multi-stream.

\section{Introduction}

The best way to reduce the fuel consumption and improve the automotive efficiency is to reduce the speed range of the internal combustion engine used [1-3]. In order to maintain the required operating speed range of the automobile, it is necessary to increase the number of overall gear ratios in the transmission. Trucks, agricultural machinery and tractors are equipped with transmissions consisting of main 3-speed or 4-speed gearbox with fixed axles of gears and a planetary splitter and/or a planetary range change unit $[4 ; 5]$. These planetary gear sets reduce the rotation speed or work in direct gear depending on the driving conditions of the machine. The splitter and range change unit are also providing up to 4 times extension of the number of gear ratios realized by transmission.

As it is known [4-7], the planetary gear set consists of three members and in dependence on their linkages has the following three operating modes.

1. Differential drive - converts the motion of two input members into the motion of one output member (summing gear) or the motion of one input member into the motion of two output members (differential gear).

2. Reducer or multiplier - when one member is fixed.

3. Blocked, rotates as a single unit with direct gear, when any two members are connected with each other.

In the transmissions of trucks and tractors, the planetary gear of the splitter or the additional gear operates only in modes 2 and 3. Mode 1 is used in planetary transmissions and in hybrids to divide or sum up the power flows of the internal combustion engine and electric motor $[4 ; 5 ; 8 ; 9]$.

In [10] it is shown that the use of the planetary gear set as a differential drive in transmissions with fixed axles of gears makes it possible to obtain simple transmissions designs, which have high efficiency and a greater number of speeds than transmissions consisting of single type of gears. A greater number of speeds is achieved by changing the mode of operation of the planetary gear set and gears with fixed axles, which allows the latter to work both separately in the one-stream mode of operation of the planetary gear set (reducer or multiplier) and in parallel with other layshaft gears in two-stream mode. Using the terminology [11], the obtained transmissions are called two-stream by the number of parallel power-flows from the input shaft to the output one. A method for the synthesis of kinematic diagrams was developed in [10], with the help of which a group of new designs of twostream transmissions were obtained [10]. 
With the use of two planetary gear sets operating as differential drives, the number of parallel power flows increases to three, which makes it possible to further improving of the functionality of the multi-stream transmissions. In addition, if in a two-stream transmission the input power-flow is divided between two paths about a half, then in three-stream transmissions this percentage decreases by a factor of 1.5 or 2 [11]. Therefore, the loads on internal gears in multi-stream transmissions are much lower, so the gear trains can be smaller in weight, overall dimensions and less complicated. The purpose of the paper is to analyse structural schemes of transmissions with mixed planetary-layshaft arrangements with three power-flows, to determine their general characteristics and to develop a method for synthesis of the kinematic diagrams of these transmissions.

\section{Synthesis of the structures}

For a visual representation of the planetary-layshaft transmission structure we used the generalized symbols of the gear trains involved in it $[6 ; 7]$. By means of generalized symbols, the planetary gear set and one or several gears with fixed axles can be represented.

By adding to the generalized symbol of planetary gear set the shifting elements that provide external couplings on the members, the planetary gear set is represented in the general case by only two generalized schemes shown in Fig 1. The schemes differ in the locations of the input and output members, and at switched-off c1, c2 and c3 determine the planetary gear set, as a summing gear, which is shown in Fig. 1a, or differential gear as shown in Fig. 1b. Two shifting elements c1, c2, c3 of the differential D cannot be switched-on at the same time.

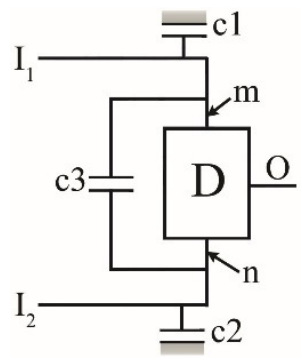

a

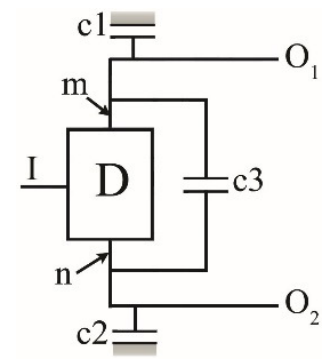

$\mathrm{b}$

Fig. 1. Generalized schemes of planetary summing gear (a) and differential gear (b): I, O - input and output members respectively; $m$ and $n$ are the members of the differential $D ; c 1$ and $c 2-$ brakes, which respectively fix the members $m$ and $n$ with the frame; $c 3$ - clutch. which links the members $m$ and $\mathrm{n}$ for differential blocking and rotation on direct gear

Similarly, one or more gears with fixed axles and shifting elements built into them, which perform different gear ratios from input shaft I to output shaft $\mathrm{O}$, are represented in Fig. 2a by the internal gearbox GB. Fig. $2 b$ shows an example of a kinematic diagram of a two-speed GB with fixed axles of gears.

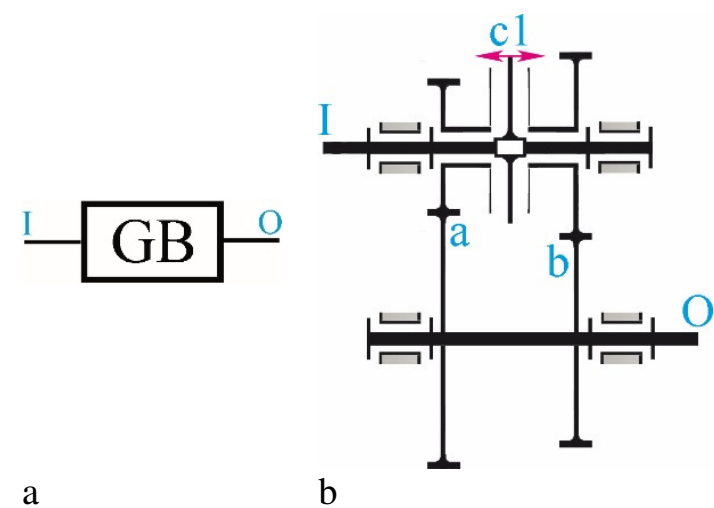

Fig. 2. Generalized symbol (a) and kinematic diagram (b) of two-speed gearbox with fixed axles of gears: I, O - input and output members respectively; $\mathrm{c} 1$ - shifting element (clutch or synchronizer), which has three working positions 
It was shown in [6] that in order to obtain three parallel power-flows with the help of two coupled planetary gear sets with three parts only 4 arrangement variants are possible. In the present paper it has been established that the integration of internal GBs into each of the power paths does not increase the number of possible arrangements. Only four different structural schemes of the three-stream planetarylayshaft transmissions are obtained as a result of the structural synthesis. The obtained structures are shown in Fig. 3.

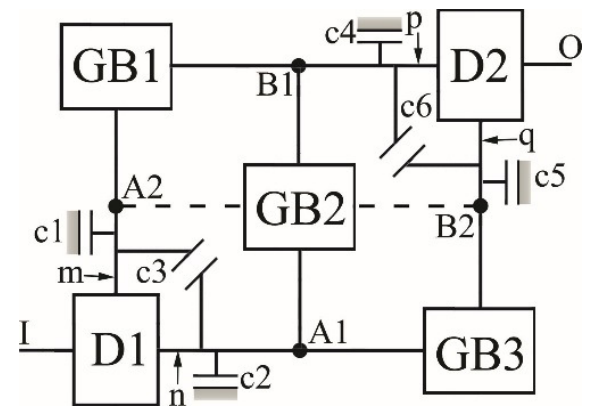

a

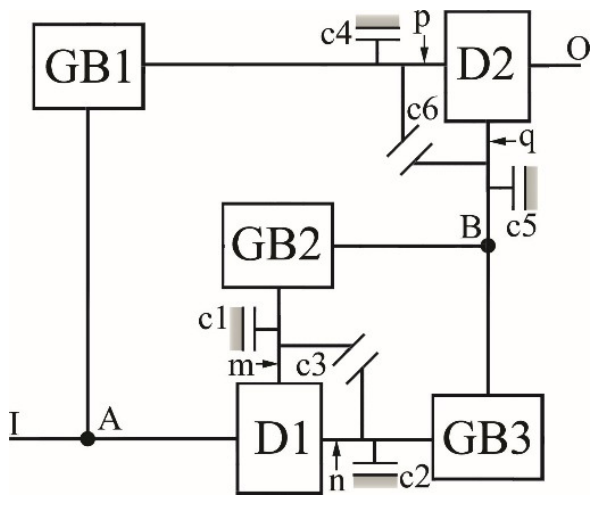

c

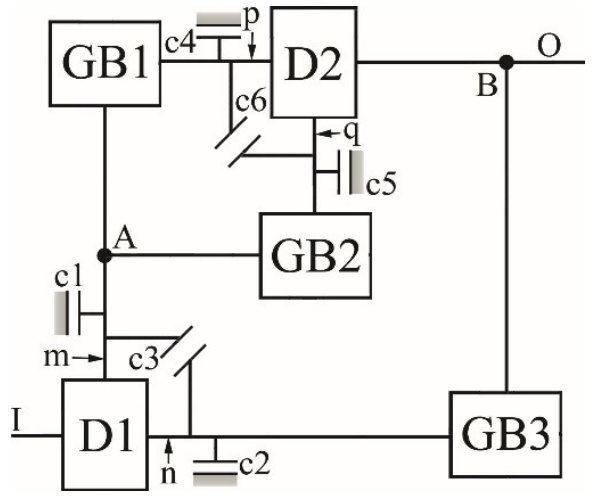

b

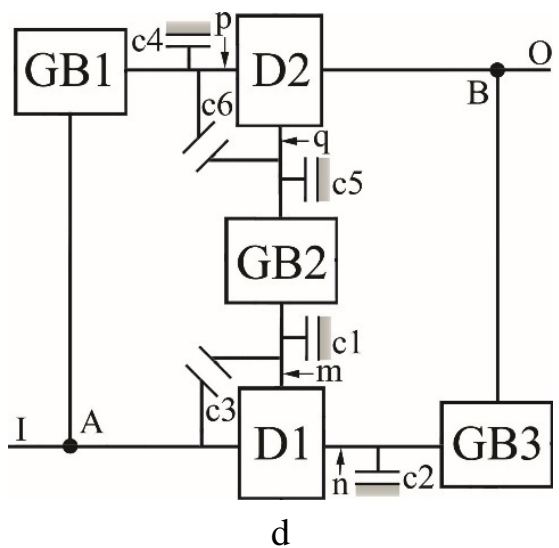

Fig. 3. Possible structures of three-stream planetary-layshaft transmissions: $\mathrm{I}, \mathrm{O}$ - input and output members respectively; $\mathrm{m}$ and $\mathrm{n}$ are the members of the differential $\mathrm{D} 1 ; \mathrm{p}$ and $\mathrm{q}$ are the members of the differential D2; $c 1, c 2$ and $c 4, c 5$ are brakes, which respectively fix members $n, m$ and $p, q$ with a frame respectively; 33 and c6 are the blocking clutches of the differentials D1 and D2 respectively

Depending on the location of the differentials, input and output members the following classification of four obtained structures is introduced:

- DD shown in Fig. 3a - the structure in which the planetary gear sets locate on the input and output;

- DDO shown in Fig. $3 b$ - the structure in which the planetary gear set locates at the input, the second one in the internal circuit, and the power-flows are summarizing on the output member O;

- IDD shown in Fig. $3 c$ - the structure in which planetary gear set locates at the output, the second one in the internal circuit, and the power-flow is distributing by the input member I.

- IDDO shown in Fig. $3 \mathrm{~d}$ - the structure in which both planetary gear sets locate in internal circuits and the power-flows are distributing by the input member I and summarizing on the output member O.

In the resulting structures each of the closed circuits on one side has a differential, and on the other the node points $\mathrm{A}$ and $\mathrm{B}$, in which the power-flows are distributing or summarizing. There are two possible options for forming an internal circuit in the structure DD shown in Fig. 3a, i.e. links between the members of internal GB2 and differentials: either with $\mathrm{m}$ and $\mathrm{q}$ (dashed lines) or with $\mathrm{n}$ and $\mathrm{p}$ (solid lines). In other structures there is only one kind of the links possible between internal 
members for forming three parallel power flows from input I to output O. The structures DD and IDDO are symmetric with respect to the internal power-flow passing through GB2. The IDD structure can be obtained from the DDO structure by replacing the input member with the output one and vice versa.

For power distribution in three paths on the three-stream mode the shifting elements in the internal gearboxes GB must be switch-on at switched-off shifting elements of the differentials. The maximum number of differentials' shifting elements (brakes c1, c2, c4, c5 and blocking clutches c3, c6) is presented on the structures in Fig. 3, which determine the possible two- and one-stream operation modes. These control elements may be absent in the specific kinematic diagrams of the transmissions, then the number of operating modes of such a transmission will accordingly decrease. Up to 4 one-stream modes for each internal GB and up to 2 two-stream modes through each pair of internal GBs are possible in each structure. In general, the number of speeds performed by a threestream transmission is defined by the expression (1).

$$
\begin{aligned}
N=N_{G B 1} \cdot l_{1}+ & N_{G B 2} \cdot l_{2}+N_{G B 3} \cdot l_{3}+N_{G B 1} \cdot N_{G B 2} \cdot l_{4}+N_{G B 1} \cdot N_{G B 3} \cdot l_{5}+ \\
& +N_{G B 2} \cdot N_{G B 3} \cdot l_{6}+N_{G B 1} \cdot N_{G B 2} \cdot N_{G B 3},
\end{aligned}
$$

where $N_{G B 1}, N_{G B 2}, N_{G B 3},-$ number of speeds in GB1, GB2, GB3 respectively;

$l_{1}, l_{2}, l_{3}$ - one-stream mode parameters for GB1, GB2, GB3 respectively;

$l_{4}$ - parameter of two-stream mode via GB1 and GB2;

$l_{5}$ - parameter of two-stream mode via GB1 and GB3;

$l_{6}$ - parameter of two-stream mode via GB2 and GB3.

Analysis of the expression (1) showed that the largest number of modes is allowed by the DD structure shown in Fig 3a. The IDDO structure shown in Fig. 3d has the least number of modes. The blocking clutches c3 and/or c6 of the planetary gear sets D1 and D2 can be switched-on for all structures in two- and one-stream modes. Therefore, these clutches should be used in the synthesis of transmissions with a greater number of speed ratios. The use of blocking clutches makes it possible to increase the efficiency of the planetary gear train because it rotates as a single unit without loss of energy $[6 ; 7]$.

\section{Kinematic diagrams synthesis}

Synthesis of kinematic diagrams is performed by replacing generalized structures with kinematic diagrams of gear trains in the selected structure. Here is an example of the synthesis a 17 -speed (including reverse gears) transmission of the structure DD. Let the internal gearboxes GB1, GB2, GB3 have equal number of speeds $N_{G B 1}=N_{G B 2}=N_{G B 3}=1$ then from the expression (1) we get the necessary number of two- and one-stream modes in the synthesized transmission:

$$
l_{1}+l_{2}+l_{3}+l_{4}+l_{5}+l_{6}=16
$$

If D1 will have brake (c1) and blocking clutch (c3), D2 will have two brakes (c4, c5) and a clutch (c6), then we get the next parameters of one- and two-stream modes: $l_{1}=2 ; l_{2}=4 ; l_{3}=4 ; l_{4}=2 ; l_{5}=2$; $l_{6}=2$. Then the sum of the $l$ values gives the required number of two- and one-stream modes in the synthesized DD transmission.

As differentials D1 and D2 we will use simple epicyclic gear sets. When connecting the sun pinion to the input shaft, the direction of rotation of the carrier will coincide with the direction of rotation of the sun, and the ring gear will rotate in the opposite direction. In this case, the gear ratio from the sun to the other two members of the epicyclic gear train will be greater than 1 . This arrangement of the planetary gear in place of the differential D1 should be considered the most effective to provide gear ratio increasing from gear to gear in the gearbox. When the planetary mechanism is blocked, it will rotate in direct gear in the same direction as the sun pinion.

The connection of the members of the epicyclic gear set located at the output of the transmission must be such that the rotation direction of either two members coincides with the rotation direction of the third one. This is only possible when the transmission output shaft is connected to the planetary carrier. 
To provide a reverse gear, one of the internal gearboxes GB must have an additional parasitic gear for reversing. Let it be GB1, it has the smallest number of one-stream modes $l_{1}=2$. Thus, the synthesized transmission will have 15 forward and 2 reverse speeds. If the ring gear of the D1 connects with GB1, then in spite of the rotation of the ring gear of D1 in the opposite direction we obtain a unidirectional rotation of all members in the transmission due to the parasitic gear in GB1. In this case at blocking D1 by the clutch c3 the reverse gears through the parasitic gear in GB1 will occur.

If each of the inner gearboxes contains one pair of fixed axles of gears, then additional gear trains for each of the GB are required for unidirectional rotation of the input and output shafts.

The result of the above considerations will be the following arrangement of the members in synthesized 17-speed transmission of DD structure.

1. The input shaft $\mathrm{I}$ is connected to the sun pinion of the D1.

2. The output shaft $\mathrm{O}$ is connected to the carrier of the $\mathrm{D} 2$.

3. The GB1 has a parasitic gear and is connected to the ring gear of the D1.

4. The GB2 is connected with the carrier of the D2.

5. The D1 has a blocking clutch $\mathrm{c} 3$, and its ring gear can be stopped by the brake $\mathrm{c} 1$.

6. The D2 has a blocking clutch c6 and two brakes c4 and c5, which stop the ring gear and the sun respectively.

7. In accordance with the structure DD in Fig. 3a, the output shafts of GB1 and GB2 are connected.

8. The output shafts of GB1, GB2 and GB3 are connected to the ring gears and the sun wheels of the D2 via countershaft gears.

The obtained transmission kinematic diagram is shown in Fig. 4.

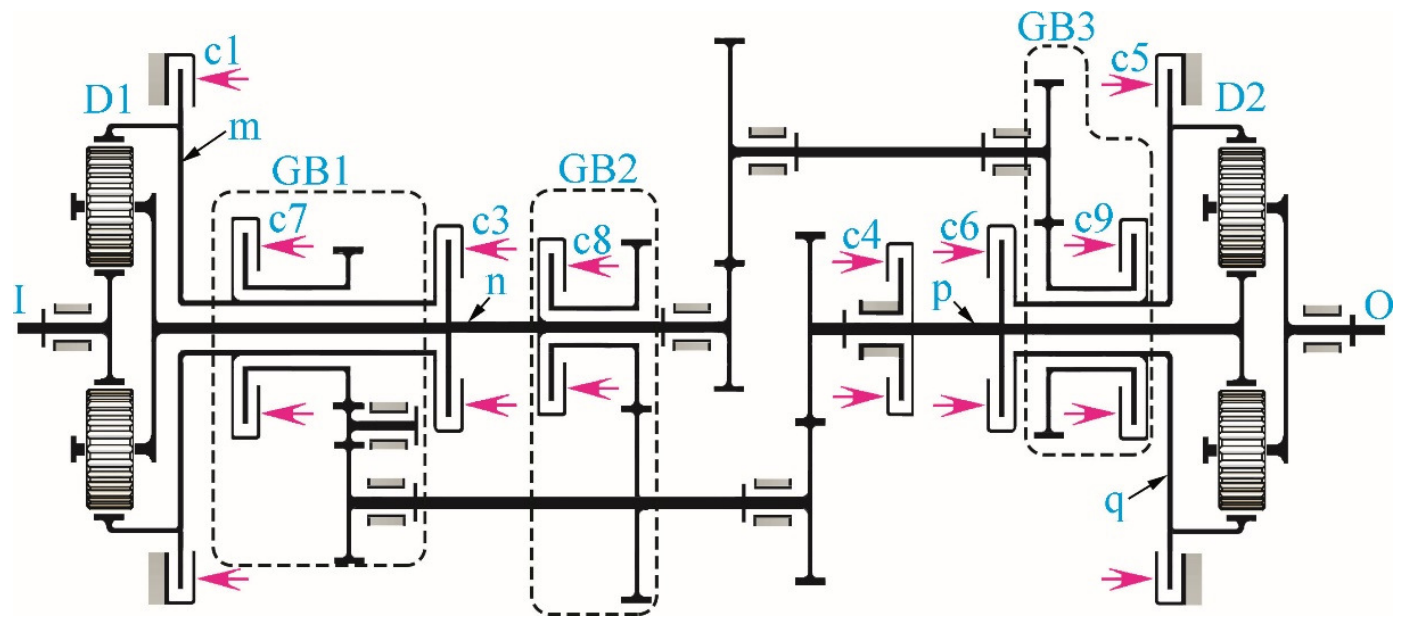

Fig. 4. 17-speed three-stream transmission design of DD structure

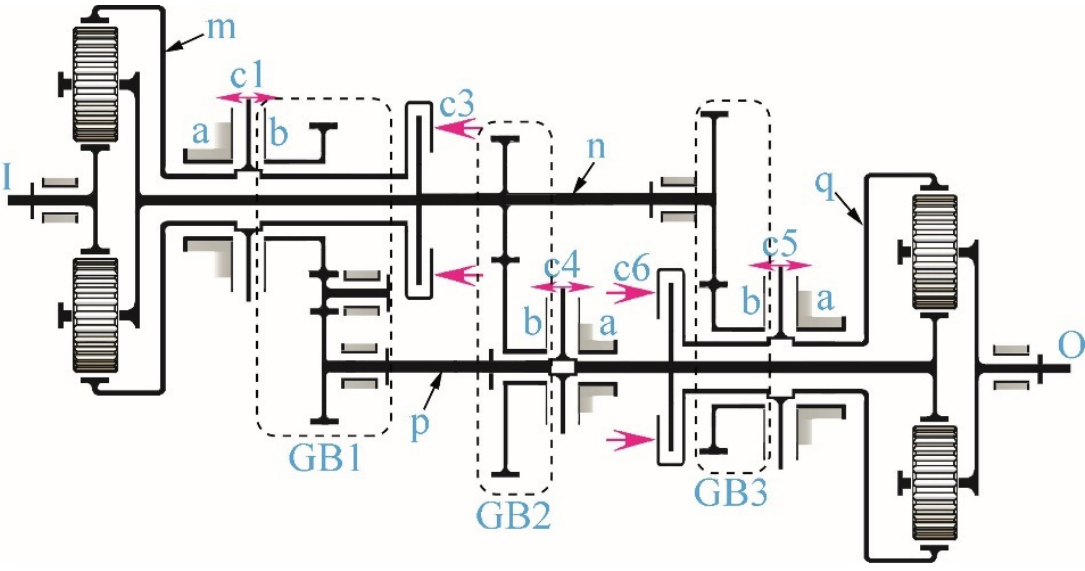

Fig. 5. 17-speed three-stream transmission design of DD structure with multidirectional rotation of $I$ and $O$ 
The clutches $\mathrm{c} 1$ and $\mathrm{c} 7, \mathrm{c} 4$ and $\mathrm{c} 8, \mathrm{c} 5$ and $\mathrm{c} 9$ of the synthesized transmission are not switched on simultaneously. Therefore, they can be combined into shifting elements with three fixed positions as in Fig. 2b. Assuming a multidirectional rotation of the input and output shafts, we obtain the kinematic diagram of the gearbox, shown in Fig. 5 [12].

In the diagram in Fig. 5, the shifting elements $\mathrm{c1}, \mathrm{c4}$, c5, at the state "a" stop the corresponding members of the planetary mechanisms, at "b" state the corresponding gears of the internal GBs operate. The operating modes (" $-"$ - one-stream; "=" - two-stream; " $\equiv "$ - three-stream) and overall gear ratios implementing by the transmission in Fig. 5 depending on the states of shifting elements are shown in Table 1 ( $\times$ - switched-on; $\mathrm{R}$ - reverse). The overall gear ratios are obtained at the following ratios between gear wheels:

- $i_{\mathrm{D} 1}=-1.5$ ratio between the sun pinion and ring gear of the D1 assuming that the carrier is stopped;

- $i_{\mathrm{D} 2}=-2.0$ ratio between the sun pinion and ring gear of the D2 assuming that the carrier is stopped;

- $i_{\mathrm{GB} 1}=3.528$ ratio between gear wheels with the parasitic gear of GB1;

- $i_{\mathrm{GB} 2}=-1.595$ ratio between gear wheels of $\mathrm{GB} 2$;

- $i_{\mathrm{GB} 3}=-0.873$ ratio between gear wheels of GB3.

The overall gear ratio calculation formulae are presented in the right column of Table 1 .

Table 1

Operating modes and overall gear ratios implemented by the transmission shown in Fig. 5.

\begin{tabular}{|c|c|c|c|c|c|c|c|c|c|c|}
\hline \multirow{3}{*}{$\begin{array}{l}\text { Speed, } \\
\text { mode }\end{array}$} & \multicolumn{8}{|c|}{ Shifting elements condition } & \multirow{3}{*}{ Ratio } & \multirow{3}{*}{ Calculation formula } \\
\hline & \multicolumn{2}{|c|}{ c1 } & \multicolumn{2}{|c|}{\begin{tabular}{|c|} 
c4 \\
\end{tabular}} & \multicolumn{2}{|c|}{ c5 } & \multirow{2}{*}{ c3 } & \multirow{2}{*}{ c6 } & & \\
\hline & $\mathbf{a}$ & b & $\mathbf{a}$ & $\mathbf{b}$ & $\mathbf{a}$ & $\mathbf{b}$ & & & & \\
\hline $1 .=$ & & $\times$ & & $x$ & $\times$ & & & & -27.8 & $\left(i_{G B 1} i_{D 1}+i_{G B 2}\left(1-i_{D 1}\right)\right)\left(1-i_{D 2}\right)$ \\
\hline 2. - & $x$ & & & $x$ & $\times$ & & & & -12.0 & $i_{G B 2}\left(1-i_{D 1}\right)\left(1-i_{D 2}\right)$ \\
\hline 3. $=$ & & $\times$ & & $\times$ & & & & $\times$ & -9.3 & $i_{G B 1} i_{D 1}+i_{G B 2}\left(1-i_{D 1}\right)$ \\
\hline 4. $=$ & & $x$ & & & & $\times$ & & $x$ & -7.5 & $i_{G B 1} i_{D 1}+i_{G B 3}\left(1-i_{D 1}\right)$ \\
\hline $5 . \equiv$ & & & & & & & & & -6.0 & ${\underline{i_{G B 3}}}\left(i_{G B 1} i_{D 1}+i_{G B 2}\left(1-i_{D 1}\right)\right)\left(1-i_{D 2}\right)$ \\
\hline J. = & & $\times$ & & $x$ & & $x$ & & & -0.0 & $i_{G B 3}-i_{G B 2} i_{D 2}$ \\
\hline $6 .-$ & & & & $x$ & $\times$ & & $x$ & & -4.8 & $i_{G B 2}\left(1-i_{D 2}\right)$ \\
\hline 7. - & $\times$ & & & $\times$ & & & & $\times$ & -4.0 & $i_{G B 2}\left(1-i_{D 1}\right)$ \\
\hline $8 .-$ & $x$ & & $\times$ & & & $x$ & & & -3.3 & $i_{G B 3}\left(1-i_{D 1}\right)\left(i_{D 2}-1\right) / i_{D 2}$ \\
\hline & & & & & & & & & & $i_{G B 2} i_{G B 3}\left(1-i_{D 1}\right)\left(1-i_{D 2}\right)$ \\
\hline 9. = & $x$ & & & $x$ & & $x$ & & & -2.6 & $i_{G B 3}-i_{G B 2} i_{D 2}$ \\
\hline $10 .-$ & $x$ & & & & & $x$ & & $\times$ & -2.2 & $i_{G B 3}\left(1-i_{D 1}\right)$ \\
\hline 11. - & & & & $x$ & & & $x$ & $x$ & -1.6 & $i_{G B 2}$ \\
\hline & & & & & & & & & & ${\underline{i_{G B 1} i_{G B 3}}\left(1-i_{D 2}\right)}$ \\
\hline $12 .=$ & & $\times$ & & & & $x$ & $\times$ & & -1.5 & $\overline{i_{G B 3}-i_{G B 1} i_{D 2}}$ \\
\hline 13. - & & & $\times$ & & & $\times$ & $\times$ & & -1.3 & $i_{G B 3}\left(i_{D 2}-1\right) / i_{D 2}$ \\
\hline & & & & & & & & & & $\underline{i_{G B 2} i_{G B 3}\left(1-i_{D 2}\right)}$ \\
\hline $14 .=$ & & & & $\times$ & & $x$ & $\times$ & & -1.0 & $i_{G B 3}-i_{G B 2} i_{D 2}$ \\
\hline 15. - & & & & & & $x$ & $\times$ & $\times$ & -0.9 & $i_{G B 3}$ \\
\hline R1. - & & $\times$ & & & $x$ & & $\times$ & & 10.6 & $i_{G B 1}\left(1-i_{D 2}\right)$ \\
\hline R2. - & & $\times$ & & & & & $\times$ & $\times$ & 3.5 & $i_{G B 1}$ \\
\hline
\end{tabular}


As a result, a compact 17 -speed transmission with five shifting elements is obtained. Three clutches are switching-on each speed, which reduces energy losses. The transmission has great ratio spread equal to 30.9 (quotient from the division of the gear ratio of the first speed to the highest gear ratio). A small number of gear trains used allows one to state that this transmission has a high efficiency.

\section{Results and conclusions}

The more gear ratios realized by transmission, the lower the fuel consumption and better efficiency. To obtain simple designs of multi-speed transmissions it is proposed to use combined planetary-layshaft gearboxes consisting of two planetary gears and gears with fixed axes of gears. In terms of the number of parallel power transmission flows, such connection schemes of mechanisms are called three-stream. Possible structures of three-stream planetary-layshaft transmissions are obtained, according to the location of differentials, input and output members, a classification of the structures is made. The analysis of operating modes of the three-stream transmissions is carried out and the possible number of realized gear ratios is obtained. By the replacement of the structures with kinematic diagrams of specific gear trains an example of the synthesis of a 17-speed transmission is given. The synthesised transmission consists of simple gears with five control elements, it has high efficiency due to the small number of gear trains and shifting elements, switched-on each gear.

\section{Acknowledgements}

The research was supported by Russian Science Foundation, project No 17-79-10493.

\section{References}

[1] Ota H., Nozaki K., Honda A. etc. Toyota's World First 8-Speed Automatic Transmission for Passenger Cars. SAE Technical Paper No 2007-01-1101. 2007.

[2] Broge J.L. ZF's nine-speed geared for front-transverse engines. [21.01.2018]. Available at: http://articles.sae.org/11884/

[3] Dong P., Liu Y., Tenberge P. etc. Design and analysis of a novel multi-speed automatic transmission with four degrees-of-freedom. Mechanism and Machine Theory, vol. 108, 2017, pp. 83-96.

[4] Förster H.J. Automatische Fahrzeuggetriebe. Berlin, Heidelberg: Springer, 1991. 529 p. (In German).

[5] Naunheimer H., Bertsche B., Ryborz J., Novak W. Automotive Transmissions. Berlin, Heidelberg: Springer, 2011.715 p.

[6] Волков Д.П., Крайнев А.Ф. Трансмиссии строительных и дорожных машин. Справочное пособие (Transmission of construction and road machinery. Reference Manual). Moscow: Mashinostroenie, 1974, 424 p. (In Russian).

[7] Müller H.W. Die Umlaufgetriebe. Konstruktionsbücher, vol 28. Berlin, Heidelberg: Springer, 1998. (In German).

[8] Zhang X.W., Li C.T., Kum D., Peng H. Prius(+) and volt(-): Configuration analysis of powersplit hybrid vehicles with a single planetary gear. IEEE Trans. Vehicular Technology, vol. 61, No. 8, 2012, pp. 3544-3552.

[9] Yang H., Kim B., Park Y. etc. Analysis of planetary gear hybrid powertrain system part 2: Output split system. International Journal of Automotive Technology, vol. 10, 2009, pp. 381-390.

[10] Kraynev A., Salamandra K., Raghavan M. Synthesis of the Two-Stream Transmissions. In: Power Transmissions. Mechanisms and Machine Science, vol 13. Dordrecht: Springer, 2013.

[11] Антонов А.С. Силовые передачи колесных и гусеничных машин. Теория и расчет (Power transmission of wheeled and caterpillar vehicles. Theory and calculation). Second edition. Leningrad: Mashinostroenie: 1975. 480 p. (In Russian).

[12] Salamandra B.L., Salamandra K.B. Gearbox. RU Patent No 2531995. 2014. 